
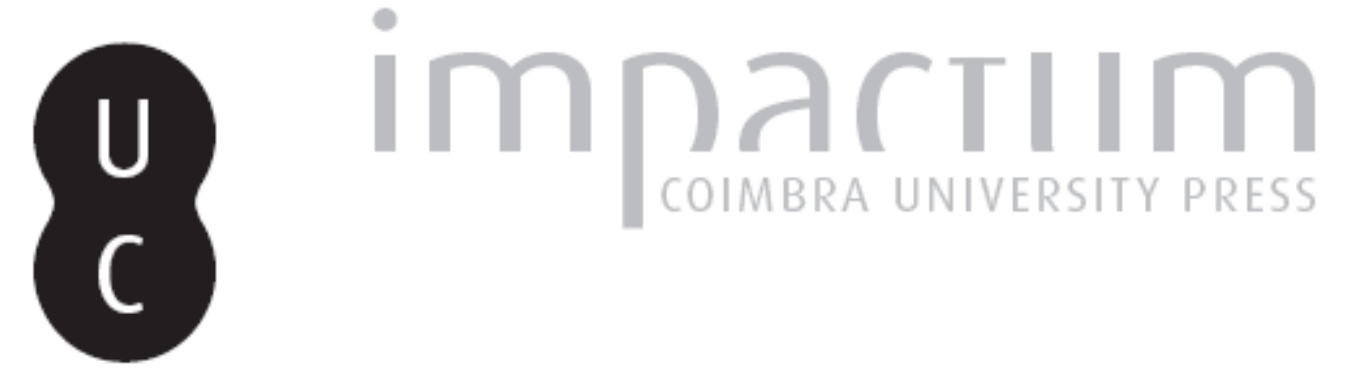

\title{
Sur de pretendues anomalies dans le Menexene de Platon
}

Autor(es): Helmer, Étienne

Publicado por: Imprensa da Universidade de Coimbra

URL persistente:

URl:http://hdl.handle.net/10316.2/42228

DOI:

DOl:https://doi.org/10.14195/2183-4105_6_5

Accessed : $\quad$ 26-Apr-2023 12:48:44

A navegação consulta e descarregamento dos títulos inseridos nas Bibliotecas Digitais UC Digitalis, UC Pombalina e UC Impactum, pressupõem a aceitação plena e sem reservas dos Termos e Condições de Uso destas Bibliotecas Digitais, disponíveis em https://digitalis.uc.pt/pt-pt/termos.

Conforme exposto nos referidos Termos e Condições de Uso, o descarregamento de títulos de acesso restrito requer uma licença válida de autorização devendo o utilizador aceder ao(s) documento(s) a partir de um endereço de IP da instituição detentora da supramencionada licença.

Ao utilizador é apenas permitido o descarregamento para uso pessoal, pelo que o emprego do(s) título(s) descarregado(s) para outro fim, designadamente comercial, carece de autorização do respetivo autor ou editor da obra.

Na medida em que todas as obras da UC Digitalis se encontram protegidas pelo Código do Direito de Autor e Direitos Conexos e demais legislação aplicável, toda a cópia, parcial ou total, deste documento, nos casos em que é legalmente admitida, deverá conter ou fazer-se acompanhar por este aviso.

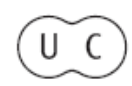




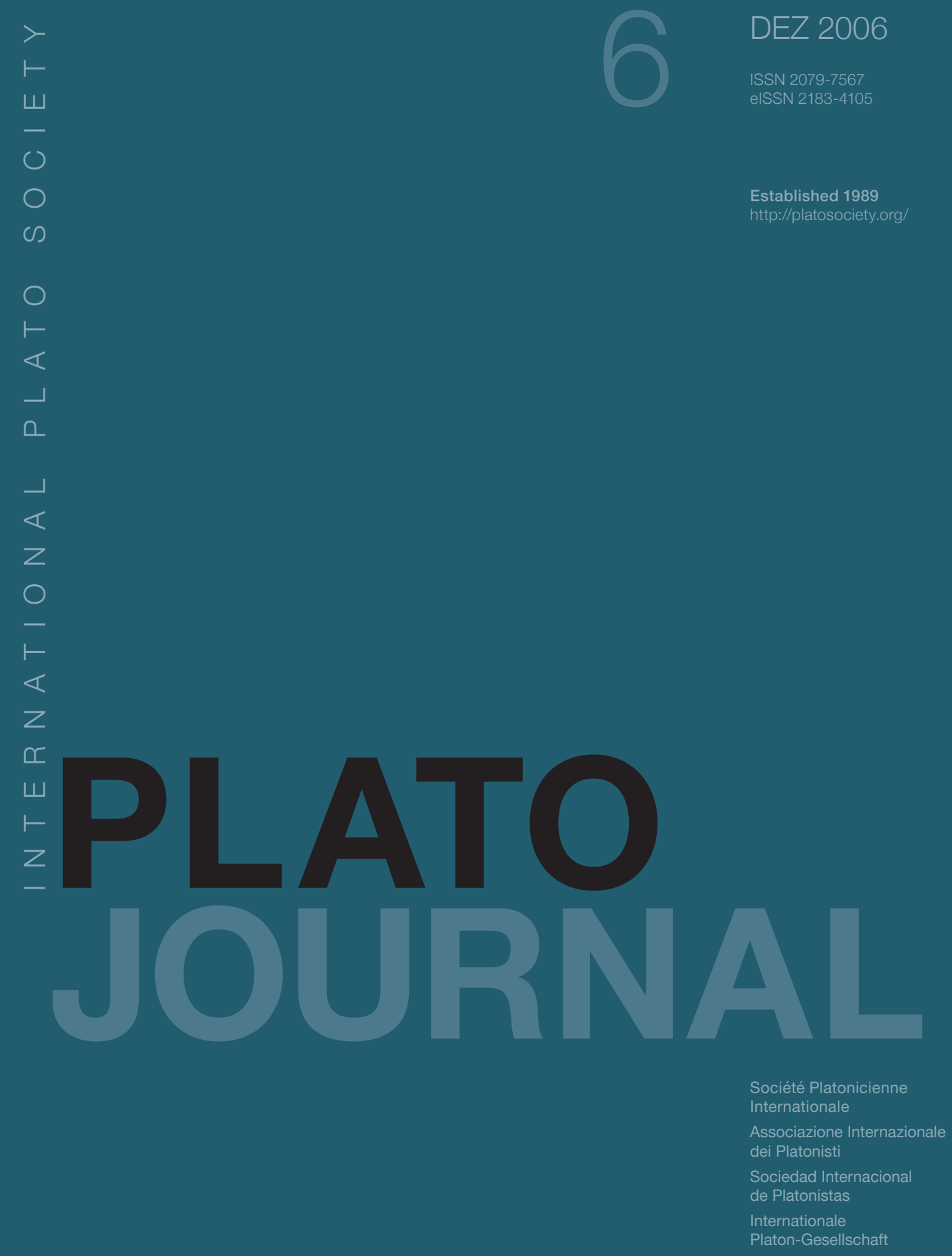




\section{SuR DE PRETENDUES ANOMALIES DANS LE MENEXENE DE Platon}

\section{Les « anomalies »}

Dans un article paru en 1991, J. Labarde relève trois anomalies dans le Ménexène de Platon ${ }^{1}:$ l'usage répété du masculin quand le locuteur se désigne luimême (autos, 246b6 ; dikaios, 246c2 ; tekmairomenos, c6 ; autos, 248e2), alors que ce discours est censé être celui d'une femme, Aspasie ; la remise en cause par Ménexène de l'identité de l'auteur de l'oraison à la fin du dialogue (hostis soi ho eipôn, « quel que soit celui qui t'a récité [cette oraison] »,249e1) alors que Socrate l'a explicitement attribuée à Aspasie dès le début (235e8-9); des anachronismes dans l'exposé des événements historiques.

Excluant l'hypothèse d'erreurs de copies, J. Labarde attribue les deux premières anomalies à l'histoire de la réception du Ménexène, dont Cicéron affirmait qu'on récitait chaque année à Athènes la partie centrale, c'est-à-dire l'oraison funèbre proprement dite ${ }^{2}$; un déclamateur public, nécessairement un homme dans l'Antiquité, aurait alors masculinisé le locuteur. Ces termes au masculin seraient donc dus à « une insertion subie par le Ménexène quand la tradition manuscrite en eut accueilli le discours masculinisé $\rangle^{3}$.

La troisième anomalie serait le corollaire des deux premières : les déclamateurs en question auraient inséré dans l'oraison des événements contemporains afin qu'elle eût un sens pour leurs auditeurs ${ }^{4}$. Ainsi s'expliquerait que Platon fasse allusion à des événements de 387, notamment le traité d'Antalcidas ou Paix du Roi (245b-e), que Socrate, mort en 399, n'a évidemment pu connaître ; ou encore qu'il évoque des événements de 403 et fasse ensuite référence au présent de l'auditeur, nécessairement ultérieur à 387, comme étant la suite logique de ces événements (243e-244b). Cette évocation du présent serait la trace d'une interpolation destinée à donner à l'oraison une tonalité et une portée contemporaines.

Comme l'a montré H. Alline, l'histoire de la transmission et de la réception matérielles des Dialogues de Platon se révèle fructueuse pour leur interprétation ${ }^{5}$. Mais elle ne doit pas pour autant se substituer à leur analyse interne, sous peine de diminuer la pertinence et l'intérêt de leur contenu. Or il nous semble que le Ménexène, ainsi que d'autres dialogues de Platon, donnent les clés de ces prétendues anomalies historiques et grammaticales relevées par J. Labarde. Loin d'être dues aux hasards de la transmission, ce passage du féminin au masculin, la remarque de Ménexène sur l'identité de l'auteur de l'oraison et les problèmes chronologiques tiennent selon nous à la stratégie critique adoptée par Platon pour exposer les effets de l'oraison, et donnent au Ménexène une portée philosophique amorcée dans ses parties dialoguées. Plus précisément, la confusion affectant le sexe et l'identité du locuteur et de l'auteur de l'oraison est autant l'origine que le résultat d'un type de discours politique fondé sur la falsification historique et sur l'édification d'une identité politique collective factice qui, comme nous le verrons, font obstacle au précepte delphique « connais-toi toi-même ».

\footnotetext{
${ }^{1}$ Labarde, J., « Anomalies dans le Ménexène de Platon », L'Antiquité classique, Louvain-La-Neuve, 60, 1991, 89-101. 
Dans le même sens, les incohérences chronologiques reflètent une conception politique qui, contrairement à la vision platonicienne de l'histoire selon laquelle le présent doit s'adosser à une norme intelligible donnant sens à l'action politique présente et future, annule l'histoire en indexant le passé aux valeurs du présent de la démocratie athénienne.

\section{L'oubli de soi}

Le discours de la cité sur ses morts produit, selon Socrate, une double identification : entre les vivants et les morts (246b), et entre les vivants eux-mêmes (234c-235a). Ce discours conduit à un oubli presque complet de soi, vouant à l'échec l'exigence socratique d'une connaissance de soi. C'est ce qu'exprime Socrate lorsqu'il prétend avec humour qu'après avoir entendu une oraison, il lui faut au minimum quatre ou cinq jours pour « se ressouvenir de lui-même » $(235 \mathrm{c} 3)$. Cette formule résume on ne peut plus clairement l'effet provoqué par l'oraison, tant par son contenu que par ses conditions d'énonciation et sa composition. Comment expliquer cet effet ?

En célébrant les vertus des soldats tombés à la guerre, la cité condamne en effet les vivants à n'être plus que l'ombre des morts, sur lesquels elle les incite à prendre modèle comme le montrera la fin de l'oraison (246a-b ; 246d-247b). Étouffant toute possibilité d'affirmation de soi par le choix réfléchi de ses propres valeurs, l'oraison ramène l'autre au même, le différent au semblable : aux yeux des étrangers qui l'accompagnent, Socrate se retrouve indifféremment paré du même prestige que celui conféré par l'oraison à la cité dans son ensemble (235b5-8). Assimilé à la cité de son temps et aux valeurs qu'elle célèbre, Socrate est donc paralysé : c'en est fini de ses paroles ou de son comportement « atopiques », qui ne manquent jamais de déconcerter ses interlocuteurs (par exemple Phèdre, 229c6 ; Gorgias, 494d1 ; Banquet, 175a10 ; République VII, 515a4). Cette assimilation de Socrate à la cité n'est pas sans ironie au regard des chefs d'inculpation retenus contre lui lors de son procès (Apologie de Socrate, 24b-c), et qui témoignent au contraire de sa dangereuse singularité. Étrangers à la cité, ces compagnons de passage sont donc aussi étrangers ou aveugles à la différence de Socrate. L'oraison funèbre fabrique ainsi une identité collective à laquelle sont subordonnés les individus : Socrate est alors assigné à une place dans la cité, tandis que sa condamnation à mort sanctionne dans l'Apologie l'impossibilité de lui en donner une. Cette identité collective factice entraîne donc la réduction à l'identique des individus, quelles que soient leur valeur morale et leurs qualités (234c-235a). L'oraison annihile toute distinction entre eux, tant en ce qui concerne la pensée que les actes. Aux antipodes de la hiérarchie des types humains et des types de vie proposée au livre IX de la République (583c-592b), le discours de la cité qui fait de la « belle mort » (246d2-3) la valeur suprême de la conduite humaine rend caduque la question la plus importante : « de quelle manière faut-il vivre ? »(Gorgias, 500c3-4), ainsi que les réflexions qu'elle implique. S'il faut mourir pour la cité, à quoi bon s'efforcer de changer de vie?

On pourrait objecter que la Prosopopée des Lois dans le Criton contredit le regard que Socrate porte ici sur Athènes : elles rappellent en effet à Socrate qu'il a toujours été libre de les convaincre de changer, ou de quitter Athènes si elles lui déplaisaient (51d-e) ; en outre, les Lois peuvent prouver que Socrate les appréciait, elles et la cité d'Athènes (52b). Celle-ci semble donc avoir respecté la singularité de Socrate, et lui semble y avoir trouvé son compte. Cette difficulté mériterait un traitement bien plus important que celui que nous pouvons lui accorder ici, mais on 
peut avancer un élément de réponse : ces portraits contrastés d'Athènes s'expliquent en partie par les problèmes différents traités par chacun de ces dialogues. Le Criton s'interroge en effet sur les conditions de participation à la vie d'une cité alors que le Ménexène examine les effets psychologiques d'un certain type de discours politique. Le Criton montre qu'obéir aux lois est la condition minimale de l'existence politique, en dépit des inconvénients que chacun peut y trouver à titre personnel, ce qui n'interdit pas de chercher à les améliorer ou à les modifier. L'universalité nécessaire à leur fonction s'érige sur la négation des intérêts subjectifs de chacun à leur obéir ou à les transgresser. Dans le Ménexène en revanche, Socrate adopte un ton polémique qui pourrait inviter à modifier les lois de la cité sur un point particulier.

Quoi qu'il en soit, ce n'est pas seulement aux yeux des étrangers que Socrate n'est pas lui-même ; par hypothèse en effet, comment pourraient-ils s'en apercevoir ? C'est principalement en lui-même qu'il se trouve comme dénaturé, affecté d'une paralysie entravant le mouvement de son âme vers la vérité. Socrate se déclare en effet « cloué sur place » (hestèka, 235a7) par l'oraison ${ }^{6}$, bien qu'il demeure suffisamment lucide pour railler après coup ce fâcheux symptôme. Cette immobilité contraste d'une part avec le reproche adressé par ses juges à Socrate : il « se mêle des affaire des autres [polupragmonei] » et est condamné « pour n'avoir pas su rester tranquille [hèsukhian] » (Apologie de Socrate, 31c5 ; 36b6), c'est-à-dire pour avoir voulu sonder les fondements des prétentions des pseudo-savants au savoir et au pouvoir, au risque de bouleverser l'ordre établi. Cette immobilité contraste d'autre part avec le mouvement de la pensée telle que la conçoit Platon : qu'il s'agisse de passer d'une hypothèse à un principe comme l'évoque le livre VI de la République (511a-d), de « s'élancer à travers le logos vers ce qu'est la chose » (République VII, 532a5-7) ou, de façon plus imagée, des propos de Socrate qui ne tiennent pas en place (Euthyphron, 11d-e), la puissance dialectique est tout entière sous le signe du mouvement parce qu'elle est par définition circulation du $\log \operatorname{sos}^{7}$.

L'oubli de soi dont Socrate est victime est dû à l'envoûtement suscité par l'oraison. Le vocabulaire de la magie présent dans ce passage fait de chaque auditeur de l'oraison, et de Ménexène en particulier, un nouvel Ulysse pris aux charmes des sirènes politiques, à cette différence prêt que le jeune homme ne fait rien pour éviter d'y succomber. Socrate se dit ensorcelé (goèteuousin, 235a2), charmé (kèloumenos, $235 \mathrm{~b} 1$ ), pris par un discours et une voix chantant comme la flûte (enaulos, 235c1). Ces termes rappellent le Protagoras, où Socrate est sous le charme des propos du sophiste (328d) comparé à Orphée (315a8-b1), aspect qu'évoque aussi l'Euthydème (289e-290a) et plus précisément encore le Sophiste (234c ; 235a ; 241b). Or si la parole socratique est elle aussi créditée d'un charme sur ses auditeurs (République II, 358b), tout sépare néanmoins ces deux manières de les entraîner : le charme de l'entretien socratique fait de l'auditeur un interlocuteur ordonnant sa réflexion à la vérité, et lui fait prendre conscience des contradictions de ses opinions au point qu'il ne sache plus ce qu'il

\footnotetext{
${ }^{6}$ Si l'on adopte la leçon des manuscrits T et W, comme É. Chambry, L. Méridier, L. Robin et R.G. Bury. J. Burnet, suivi par J. Lamb, adopte la leçon du manuscrit F « exestèka » qui signifie « je suis transformé », au sens d'un gain de valeur que confirme la suite du passage. En faveur de la première hypothèse, N. Loraux souligne que cet emploi d'hestanai au sens de « rester sur place, sans bouger » est attesté depuis Homère et très prisé de la comédie ancienne, surtout d'Aristophane ; "Socrate contrepoison de l'oraison funèbre. Enjeu et signification du Ménexène », L'Antiquité classique, 43, 1974, n. 68,181 .

${ }^{7}$ Dixsaut, M., Le Naturel philosophe. Essai sur les dialogues de Platon, Paris, Vrin, Tradition de la pensée classique, 1985, 27 et 30-31.
} 
pense, comme Euthyphron en fait l'expérience (Euthyphron, 11b6-7) ; au contraire, l'oraison ensorcelle pour imposer ce qu'on doit penser, comme l'indiquent les sept occurrences de l'impératif « il faut se souvenir» $(239 \mathrm{c} 5 ; \mathrm{d} 3 ; 241 \mathrm{e} 2 ; 243 \mathrm{c} 7 ; 244 \mathrm{a} 3-$ $4 ; 246 \mathrm{~b} 2 ; 247 \mathrm{~d} 8)$. Ce « devoir de mémoire » s'oppose ainsi à l'inventivité que l'entreprise dialectique suppose et qu'elle doit produire chez l'auditeur (Politique, 286e-287a). En somme, l'oraison est incompatible avec l'hypothèse d'un naturel philosophe, c'est-à-dire d'une singularité pensante capable de s'engager dans un dialogue et de soumettre ses propres croyances à l'épreuve de la vérité sans tenir compte de l'opinion de la foule (Gorgias, 474b). Elle ne s'accommode que d'une reprise des mêmes contenus de pensée, et demeure indifférente à qui l'énonce, ce que signalent les « règles » de composition auxquelles elle obéit et qui sont destinées à en faciliter la reproduction. Qu'il s'agisse du réemploi de poncifs - ce que Socrate nomme des « rognures » d'oraisons antérieures (236b6) - comme ceux de l'autochtonie (237b238 b) ou de l'égalité démocratique $(238 b-239 a)^{8}$, de la juxtaposition grossière des événements mécaniquement introduits par les formules meta de touto $(238 \mathrm{a} 7 ; 241 \mathrm{~d} 1$; $242 \mathrm{a} 6$; 244b3) et meta de tauta $(242 \mathrm{c} 2 ; 243 \mathrm{~d} 7)$, d'effets rhétoriques éculés fondés sur la répétition d'un même mot ou sur des allitérations lourdement appuyées (par exemple : polemon / polemein / epolemei, 245b1-2 ; dia pantos pasan pantôs prothumian peirasthe, 247a2-3), de l'introduction d'une maxime traditionnelle - « rien de trop » - énoncée comme une formule rituelle (247e), tous ces procédés signalent que l'oraison sollicite davantage des facultés de mémoire que d'inventivité chez l'auteur comme chez l'auditeur. Encore s'agit-il d'une mémoire « morte », colportant un contenu presque toujours identique et « tout prêt» $(235 \mathrm{~d})$, et non de cette réminiscence en quête de vérités que l'âme possède virtuellement et qui suppose « d'être courageux et de chercher sans craindre la fatigue » (Ménon, 81d3-4). Les conditions de possibilité d'une oraison sont incompatibles avec celles du dialogue, a fortiori avec celles de la dialectique : l'oraison ne s'invente pas, elle se répète.

On comprend donc pourquoi Socrate déclare qu'il ne pourrait rien tirer de luimême s'il lui fallait prononcer une oraison (236a8). Ce type de discours rend impossible de se conformer au « connais-toi toi-même » évoqué dans l'Alcibiade (124a8) comme condition du soin à apporter à soi-même et à son âme. Que se connaître soi-même signifie rechercher son identité propre ou prendre la mesure de sa propre ignorance (Charmide, 167a-b) ${ }^{9}$, les impératifs qui figurent dans l'exhortation et la consolation finales de l'oraison (246d-249c) et qui imposent aux individus des normes extérieures de comportement et de croyance expriment l'idéologie de la cité : celle-ci ne peut s'accommoder d'une réappropriation des individus par eux-mêmes. Elle encourage au contraire leur passivité intellectuelle et pratique ${ }^{10}$ : elle leur dit ce qu'il faut savoir, ce qui leur évite de se demander ce qu'ils ne savent pas.

Le passage du féminin dans le prétendu discours original d'Aspasie à un masculin dans sa reprise par Socrate témoigne donc de cet oubli de soi dont l'oraison est la cause. Certes Socrate a dû masculiniser les termes se rapportant au locuteur pour réciter le discours. Mais cette transformation signifie aussi que l'oraison, étant le

\footnotetext{
8 Pour la comparaison des occurrences de ces thèmes dans les oraisons de la période classique, voir J.-E. Ziolowski Thucydides and the tradition of funeral speeches at Athens, Salem, Arno Press, 1981.

9 Sur la signification du précepte « connais-toi toi-même », voir L.-A. Dorion, Platon. Charmide. Lysis, Paris, 2004, Flammarion, 53-68.

${ }^{10}$ Coventry, L., « Philosophy and Rhetoric in the Menexenus », Journal of Hellenic Studies, 109, 1989, 6.
} 
discours de toute la cité, n'est en propre celui de personne, c'est-à-dire d'aucune âme particulière. À la différence du Banquet où il rapporte son dialogue avec Diotime sans s'effacer derrière elle et en conservant la distance dialogique nécessaire à l'échange des questions et des réponses, Socrate répète ici un discours funèbre qui, précisément parce que son efficacité est suspendue à cette répétition et parce qu'il doit être le discours de tous les membres de la cité, annihile toute différence entre eux, y compris sexuelle. Celle-ci s'efface derrière le thème de l'origine autochtone, commune à tous. Ainsi, si Ménexène s'étonne qu'une femme puisse composer de tels discours (249d4-5), puis laisse immédiatement après planer un doute mêlé d'indifférence sur l'identité de l'auteur de cette oraison (hostis soi ho eipôn, « quel que soit celui qui te l'a récitée », $249 \mathrm{e} 1$ ), ce n'est pas seulement en raison des préjugés culturels de l'époque mettant les femmes à l'écart de la vie politique. C'est qu'il a sans doute remarqué ce qu'il y a d'incongru à ce qu'une femme expose le thème de l'autochtonie et affirme que c'est de la terre que naissent les hommes (237e5-7) : Aspasie se serait en quelque sorte oubliée elle-même en tant que femme, signalant ainsi que l'oraison suppose et produit cet oubli de soi éprouvé par Socrate. Vivants et morts, vivants entre eux, hommes et femmes : en promouvant la « belle mort » au rang de règle éthique suprême, l'oraison passe sous silence des différences anthropologiques que les régimes politiques idéaux de Platon s'efforcent au contraire de régler pour produire une cité juste.

\section{L'oubli de l'histoire}

On a pu voir dans l'anachronisme consistant à faire parler Socrate d'événements ultérieurs à sa mort la transposition d'un passage du livre X de l'Odyssée (v. 492 sq.), faisant de Socrate une sorte de Tirésias dont la lucidité tranche avec l'ignorance où sont plongées les ombres des défunts ; Socrate, parlant depuis la mort, jouerait envers les vivants le rôle que son démon a joué pour lui durant sa vie, éclairant avec ironie les Athéniens sur leur condition ${ }^{11}$. Or si la mort est effectivement le point de vue d'où Platon fait parler Socrate, c'est aussi selon nous pour signaler que la politique et la rhétorique athéniennes arrêtent l'histoire, et entravent ainsi tout projet de réforme intellectuelle et politique.

Englobés dans un même éloge (235a3-6), les vivants et les morts ne forment plus qu'un tout indistinct d'un point de vue non seulement synchronique, dans le présent de l'audition de l'oraison, mais aussi diachronique. L'oraison rapporte en effet les vicissitudes de l'histoire d'Athènes à d'immuables valeurs auxquelles elle serait demeurée fidèle (245c), dont l'égalité (239a) en particulier, et que les citoyens sont sommés de perpétuer invariablement : «En souvenir [des morts], tout homme doit donc exhorter leurs descendants, comme on le fait à la guerre, à ne pas abandonner le poste de ses ancêtres et à ne pas battre en retraire en cédant à la lâcheté » (246b2-5). Or cette volonté explicite de perpétuer une Athènes toujours semblable à elle-même instaure une sorte d'éternel présent expliquant le statut des anachronismes apparents relevés par J. Labarde.

Si l'on entend en effet par histoire non pas la simple distinction de l'avant et de l'après, mais la recherche d'un sens dans le cours changeant des événements, la succession chronologique que scandent les meta de touto ou les meta de tauta n'exprime précisément aucun mouvement historique : elle le nie au contraire en étayant

\footnotetext{
11 Dean-Jones, L., « Menexenus, son of Socrates », Classical Quarterly, 45, 1995, 56-57 ; Rosenstock, B., « Socrates as revenant. A reading of the Menexenus », Phoenix, 48, 1994, 341.
} 
bien plutôt l'immuabilité dont Athènes se targue dans cette oraison. Parce qu'elle est soumise aux contraintes idéologiques d'une cité prétendant n'avoir jamais trahi ses valeurs fondatrices, l'oraison célèbre ici un présent contaminant toutes les dimensions du temps. Les « anachronismes » relevés par J. Labarde ne sont pas seulement imputables à la falsification générale dont les événements historiques de cette oraison font l'objet ${ }^{12}$ : ce que l'oraison dément encore plus profondément, c'est la notion d'histoire elle-même. C'est pourquoi faire parler Socrate d'un événement qu'il n'a pu connaître, et faire du présent de l'auditeur le prolongement immédiat des événements de 403, ce n'est pas simplement allonger la série des mensonges, et c'est encore moins la trace d'un remaniement ultérieur de la part d'un déclamateur peu soucieux de vraisemblance historique ; c'est au contraire pour Platon le moyen de montrer qu'une invariable Athènes démocratique, érigée en étalon de tous les événements, réduit la possibilité de réfléchir sur un régime idéal différent d'elle, et promeut le fait présent au rang de norme. Relever l'anachronisme, c'est vouloir réintroduire l'histoire dans un discours qui la refuse. On pourra objecter qu'en présentant la démocratie comme le régime originel et jamais démenti d'Athènes, Socrate introduit pourtant un hôs ta polla qui semble annuler, ou tout au moins nuancer, le ton aei khronon précédent (238c6-7), comme si l'histoire reparaissait discrètement au moment même où elle est niée. Or cette rectification fait partie de la stratégie critique de Platon : 1'oraison étant par nature non dialogique, le seul moyen de créer une distance critique pour percevoir ce que Platon y dénonce consiste à la mimer en y introduisant des détails qui perturbent sa rhétorique tendancieuse et ses effets affectifs.

Quand Platon, pour sa réflexion politique, convoque l'histoire, celle-ci reçoit au contraire son intelligibilité de la norme d'un régime politique parfait. Le présent n'est plus alors le critère d'intelligibilité d'une histoire qu'en réalité il abolit; il est au contraire évalué à l'aune d'une norme intelligible idéale qui donne sens à l'histoire. Le livre III des Lois en est la parfaite illustration. Deux « espèces mères » de régimes politiques y sont définies : le gouvernement d'un seul, ou monarchie, dont les Perses sont l'exemple type, et le gouvernement du peuple, ou démocratie, représenté typiquement par le régime des Athéniens (III, 693d2-7) ; " les autres, à peu près tous sont [...] des variétés issues de ces deux-là » (693d6-7). En dépit des apparences, cette typologie sommaire ne repose pas sur le critère du nombre des gouvernants.

L'extension du souverain est en réalité subordonnée à la valeur dominante du régime : la soumission à l'égard de la source unique du pouvoir pour les Perses, et la liberté pour Athènes. D'après l'Athénien, les critères du bon régime politique sont la liberté, l'amitié et l'intelligence, conjuguées selon une juste mesure (693d7-e3; 701d7-10). Pour y parvenir, une cité doit donc participer des deux espèces mères, et instaurer un équilibre ou une " amitié » entre servitude et liberté. Avec le temps, le pouvoir monarchique a dégénéré en despotisme chez les Perses, c'est-à-dire en excès de servitude ; et chez les Athéniens, la liberté, conçue comme servitude volontaire à l'égard de la loi, a dégénéré en licence, c'est-à-dire en excès de liberté. Chacun de ces deux régimes a donc perdu l'amitié nécessaire à son unité (693e5-702b1). Comparés à leurs contemporains perses et athéniens, les régimes actuels de Sparte et de Crète sont plus proches de cet équilibre (693e7-694a1).

La restriction de la diversité empirique et historique des régimes à deux espèces caractérisées par un principe politique propre fournit alors une grille de lecture de

\footnotetext{
${ }^{12}$ Méridier, L., Platon, CEuvres complètes, Tome V, Ière partie, Paris, Les Belles Lettres, C.U.F., 1931, 64.
} 
l'histoire des cités, à la fois descriptive et normative. Selon leur degré de participation à ces deux sortes de régimes que représentent celui de l'ancienne Athènes et celui de l'ancienne Perse, Clinias et Mégille peuvent mesurer l'évolution des cités et la juger en termes d'amélioration ou de détérioration. Dans la perspective des Lois, à savoir la fondation d'une cité jusque dans ses aspects les plus concrets, les exemples historiques ne peuvent s'avérer utiles qu'à condition d'avoir été soumis à une norme d'intelligibilité rationnelle permettant de porter un regard critique sur les cités passées et présentes, afin de construire à l'avenir la meilleure cité possible. En ce sens, la classification des régimes exposée dans les Lois permet de porter un jugement critique sur l'histoire et de lui donner un sens, non comme une destination globale enfin révélée dont il faudrait poursuivre le cours, mais comme le substrat d'une analyse critique destinée à orienter l'édification du régime juste sur des bases rationnelles justifiant le contenu concret des lois. Ce passage, inscrit dans le projet général de la fondation de la cité des Magnètes, constitue donc une histoire politique envisagée du point de vue de la rationalité normative des lois, sur le fondement de laquelle doit s'édifier la nouvelle cité.

Alors que ce passage des Lois prend acte de changements historiques dont il a élaboré les conditions d'intelligibilité à des fins politiques, l'oraison du Ménexène interdit toute histoire d'Athènes et par conséquent toute perspective de changement politique : on ne saurait donc y trouver d'anachronismes à proprement parler. On ne sait d'ailleurs pas dans quelles circonstances précises de la politique extérieure athénienne le discours d'Aspasie doit être prononcé : peu importe, puisque l'oraison dessine le portrait d'une Athènes immuable. En déclarant que les oraisons funèbres lui font perdre temporairement la conscience de l'endroit où il se trouve et lui donnent l'illusion de se trouver sur l'île des Bienheureux (235c), Socrate exprime cette annulation de l'histoire sur le ton de la plaisanterie. L'évocation de la demeure des âmes justes en général, et des philosophes en particulier (Gorgias, 523a-527a ; République VII, 540b), souligne la puissance mystificatrice d'un discours politique conférant au présent la valeur d'un absolu atemporel. Dans cette Athènes ironiquement identifiée au paradis, le philosophe n'a d'autre choix que de parodier pour se faire entendre, au risque d'être pris au sérieux.

\section{Conclusion}

La mystification est à son comble quand le mythe se fait passer pour l'histoire et fait oublier qu'il est un mythe. Or telle est la puissance de l'oraison : elle est productrice d'oubli sous couvert d'impératif de souvenir ${ }^{13}$. Rapportées à cette fonction idéologique, les prétendues anomalies du Ménexène prennent toute leur signification. Les deux premières doivent être comprises comme l'expression paroxystique de l'oubli de soi dont Socrate dit faire l'épreuve et qui guette Ménexène : l'oraison funèbre fait disparaître jusqu'à la différence anthropologique élémentaire du féminin et masculin parce qu'elle célèbre la communauté des vivants en l'alignant sur la masse indistincte des morts. En se présentant comme le discours de tous les citoyens unis et individuellement annulés dans une même soumission aux valeurs de la démocratie athénienne, elle ne peut donc être le discours de personne. Dans le prolongement de cette œuvre de mystification, la troisième « anomalie » est « sensationnelle et intentionnelle $»^{14}$ : elle dénonce un oubli de l'histoire qui, en instaurant un éternel

\footnotetext{
13 Loraux, N., art.cit. 182.

14 Dodds, E.R., Plato. Gorgias, [1959], Oxford, Clarendon Press, 1990, 24.
} 
E. Helmer 8 sur 8

présent, a pour fonction d'empêcher la pensée et la réalisation d'un autre régime politique que celui du moment.

Étienne HELMER

Lycée Louise Weiss 